\title{
PRIMARY BLACK HOLE SPIN IN OJ 287 AS DETERMINED BY THE GENERAL RELATIVITY CENTENARY FLARE
}

M. J. Valtonen ${ }^{1,2}$, S. Zola ${ }^{3,4}$, S. Ciprini ${ }^{5,6}$, A. Gopakumar ${ }^{7}$, K. Matsumoto $^{8}$, K. Sadakane ${ }^{8}$, M. Kidger ${ }^{9}$, K. Gazeas $^{10}$, K. Nilsson ${ }^{1}$, A. Berdyugin ${ }^{2}$, V. Piirola ${ }^{1,2}$, H. Jermak ${ }^{11}$, K. S. Baliyan ${ }^{12}$, F. Alicavus ${ }^{13,14}{ }^{14}$ D. Boyd ${ }^{15}$, M. Campas Torrent ${ }^{16}$, F. Campos ${ }^{17}$, J. Carrillo Gómez ${ }^{18}$, D. B. Caton ${ }^{19}$, V. Chavushyan ${ }^{20}$, J. Dalessio ${ }^{21}$, B. Debski ${ }^{3}$, D. Dimitrov ${ }^{22}$, M. Drozdz ${ }^{4}$, H. ER $^{23}$, A. Erdem ${ }^{13,14}$, A. Escartin Pérez ${ }^{24}$, V. Fallah Ramazani ${ }^{2}$, A. V. FilippenKo ${ }^{25}$, S. Ganesh ${ }^{12}$, F. Garcia ${ }^{26}$, F. Gómez Pinilla ${ }^{27}$, M. Gopinathan ${ }^{28}$, J. B. Haislip ${ }^{29}$, R. Hudec $^{30,31}$, G. Hurst ${ }^{32}$, K. M. Ivarsen ${ }^{29}$, M. JelineK ${ }^{30}$, A. Joshi $^{28}$, M. Kagitani ${ }^{33}$, N. Kaur ${ }^{12}$, W. C. Keel ${ }^{34}$, A. P. LaCluyze ${ }^{29}$, B. C. LeE ${ }^{35,36}$, E. Lindfors ${ }^{2}$, J. Lozano de Haro ${ }^{37}$, J. P. Moore ${ }^{29}$, M. Mugrauer ${ }^{38}$, R. Naves Nogues ${ }^{16}$, A. W. Neely ${ }^{39}$, R. H. Nelson ${ }^{40}$, W. Ogloza ${ }^{4}$, S. Okano ${ }^{33}$, J. C. Pander ${ }^{28}$, M. Perri ${ }^{5,41}$,

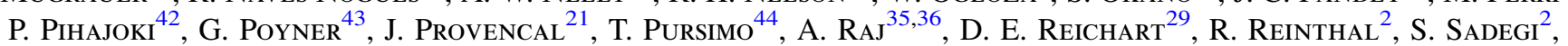
T. Sakanoi ${ }^{33}$, J.-L. Salto González ${ }^{45}$, Sameer ${ }^{12}$, T. Schweyer ${ }^{46,47}$, M. SiwaK ${ }^{4}$, F. C. Soldán Alfaro ${ }^{48}$, E. Sonbas ${ }^{49}$, I. Steele ${ }^{11}$, J. T. Stocke ${ }^{50}$, J. Strobl ${ }^{30}$, L. O. Takalo ${ }^{2}$, T. Tomov ${ }^{51}$, L. Tremosa EsPasa ${ }^{52}$, J. R. Valdes ${ }^{20}$, J. Valero Pérez ${ }^{53}$, F. Verrecchia ${ }^{5,41}$, J. R. WebB ${ }^{54}$, M. Yoneda ${ }^{55}$, M. Zejmo ${ }^{56}$, W. Zheng ${ }^{25}$, J. Telting ${ }^{44}$, J. SAario ${ }^{44}$, T. ReynOlds ${ }^{44}$, A. Kvammen $^{44}$, E. Gafton ${ }^{44}$, R. Karjalainen ${ }^{57}$, J. Harmanen ${ }^{2}$, and P. Blay ${ }^{58}$

${ }^{1}$ Finnish Centre for Astronomy with ESO, University of Turku, Turku, Finland; mvaltonen2001@yahoo.com

2 Tuorla Observatory, Department of Physics and Astronomy, University of Turku, Turku, Finland

${ }^{3}$ Astronomical Observatory, Jagiellonian University, ul. Orla 171, Cracow PL-30-244, Poland

${ }^{4}$ Mt. Suhora Astronomical Observatory, Pedagogical University, ul. Podchorazych 2, PL30-084 Cracow, Poland 5 Agenzia Spaziale Italiana (ASI) Science Data Center, I-00133 Roma, Italy

${ }^{6}$ Istituto Nazionale di Fisica Nucleare, Sezione di Perugia, I-06123 Perugia, Italy

7 Department of Astronomy and Astrophysics, Tata Institute of Fundamental Research, Mumbai 400005, India

${ }^{8}$ Astronomical Institute, Osaka Kyoiku University, 4-698 Asahigaoka, Kashiwara, Osaka 582-8582, Japan

${ }^{9}$ Herschel Science Centre, ESAC, European Space Agency, 28691 Villanueva de la Cañada, Madrid, Spain

${ }^{10}$ Department of Astrophysics, Astronomy and Mechanics, National \& Kapodistrian University of Athens, Zografos GR-15784, Athens, Greece

${ }^{1}$ Astrophysics Research Institute, Liverpool John Moores University, IC2, Liverpool Science Park, Brownlow Hill L3 5RF, UK

2 Physical Research Laboratory, Ahmedabad 380009, India

${ }^{13}$ Department of Physics, Faculty of Arts and Sciences, Canakkale Onsekiz Mart University, TR-17100 Canakkale, Turkey

${ }^{14}$ Astrophysics Research Center and Ulupinar Observatory, Canakkale Onsekiz Mart University, TR-17100, Canakkale, Turkey

${ }^{15}$ 5, Silver Lane, West Challow, Wantage, Oxon, OX12 9TX, UK

${ }^{16} \mathrm{C} /$ Jaume Balmes No 24, E-08348 Cabrils, Barcelona, Spain

${ }_{18} \mathrm{C} / \mathrm{Riera}, 1,1^{o} 3^{a}$ Barcelona, Spain

${ }^{18}$ Carretera de Martos 28 primero Fuensanta, Jaen, Spain

${ }^{19}$ Dark Sky Observatory, Dept. of Physics and Astronomy, Appalachian State University, Boone, NC 28608, USA

${ }^{20}$ Instituto Nacional de Astrofisica, Óptica y Electrónica, Apartado Postal 51-216, 72000 Puebla, México

${ }^{21}$ University of Delaware, Department of Physics and Astronomy, Newark, DE 19716, USA

22 Institute of Astronomy and NAO, Bulg. Acad. Sc., 72 Tsarigradsko Chaussee Blvd., 1784 Sofia, Bulgaria

${ }^{23}$ Department of Astronomy and Astrophysics, Ataturk University, Erzurum, 25240, Turkey

${ }^{24}$ Aritz Bidea No 84 B (48100) Mungia Bizkaia, Spain

25 Department of Astronomy, University of California, Berkeley, CA 94720-3411, USA

Muñas de Arriba La Vara, Valdés (MPC J38) 33780 Valdés, Asturias, Spain

${ }^{27}$ C/ Concejo de Teverga 9, 1 C, E-28053 Madrid, Spain

28 Aryabhatta Research Institute of Observational Sciences (ARIES), Nainital, 263002 India

${ }^{29}$ University of North Carolina at Chapel Hill, Chapel Hill, NC 27599, USA

${ }^{30}$ Astronomical Institute, The Czech Academy of Sciences, 25165 Ondřejov, Czech Republic

${ }^{31}$ Czech Technical University in Prague, Faculty of Electrical Engineering, Prague, Czech Republic

3216 Westminster Close, Basingstoke, Hampshire RG22 4PP, UK

33 Planetary Plasma and Atmospheric Research Center, Tohoku University, Sendai, Japan

${ }^{34}$ Department of Physics and Astronomy and SARA Observatory, University of Alabama, Box 870324, Tuscaloosa, AL 35487, USA

${ }^{35}$ Korea Astronomy and Space Science Institute, 776, Daedeokdae-Ro, Youseong-Gu, 305-348 Daejeon, Korea

${ }^{36}$ Korea University of Science and Technology, Gajeong-Ro Yuseong-Gu, 305-333 Daejeon, Korea

${ }^{37}$ Partida de Maitino, pol. 2 num. 163 (03206) Elche, Alicante, Spain

38 Astrophysikalisches Institut und Universitäts-Sternwarte, Schillergäßchen 2-3, D-07745 Jena, Germany ${ }^{39} \mathrm{NF} /$ Observatory, Silver City, NM 88041, USA

${ }^{40} 1393$ Garvin Street, Prince George, BC V2 M 3Z1, Canada

${ }^{41}$ INAF-Osservatorio Astronomico di Roma, via Frascati 33, I-00040 Monteporzio Catone, Italy

${ }^{42}$ Department of Physics, University of Helsinki, P.O. Box 64, FI-00014 Helsinki, Finland

43 BAA Variable Star Section, 67 Ellerton Road, Kingstanding, Birmingham B44 0QE, UK

${ }^{44}$ Nordic Optical Telescope, Apartado 474, E-38700 Santa Cruz de La Palma, Spain

45 Observatori Cal Maciarol mòdul 8. Masia Cal Maciarol, camí de l'Observatori s/n, E-25691 Àger, Spain

${ }_{46}$ Max Planck Institute for Extraterrestrial Physics, Giessenbachstrasse, D-85748 Garching, Germany

${ }^{47}$ Technische Universität München, Physik Department, James-Franck-Str., D-85748 Garching, Germany ${ }^{48} \mathrm{C} /$ Petrarca $61^{a}$, E-41006 Sevilla, Spain

49 University of Adiyaman, Department of Physics, 02040 Adiyaman, Turkey

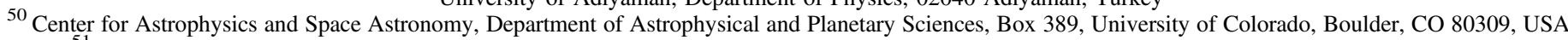

${ }^{51}$ Centre for Astronomy, Faculty of Physics, Astronomy and Informatics, Nicolaus Copernicus University, ul. Grudziadzka 5, 87-100 Torun, Poland

${ }^{52} \mathrm{C} /$ Cardenal Vidal i Barraquee No 3, E-43850 Cambrils, Tarragona, Spain

${ }^{53} \mathrm{C} /$ Matarrasa, 1624411 Ponferrada, León, Spain

${ }^{54}$ Florida International University and SARA Observatory, University Park Campus, Miami, FL 33199, USA 


\author{
${ }^{55}$ Kiepenheuer-Institut fur Sonnenphysic, D-79104 Freiburg, Germany \\ ${ }^{56}$ Janusz Gil Institute of Astronomy, University of Zielona Góra, Szafrana 2, PL-65-516 Zielona Góra, Poland \\ ${ }^{57}$ Isaac Newton Group of Telescopes, Apartado 321, E-38700 Santa Cruz de La Palma, Spain \\ ${ }^{58}$ IAC-NOT, C/Via Lactea, S/N, E-38205, La Laguna, Spain \\ Received 2016 February 12; accepted 2016 February 24; published 2016 March 10
}

\begin{abstract}
OJ 287 is a quasi-periodic quasar with roughly 12 year optical cycles. It displays prominent outbursts that are predictable in a binary black hole model. The model predicted a major optical outburst in 2015 December. We found that the outburst did occur within the expected time range, peaking on 2015 December 5 at magnitude 12.9 in the optical $R$-band. Based on Swift/XRT satellite measurements and optical polarization data, we find that it included a major thermal component. Its timing provides an accurate estimate for the spin of the primary black hole, $\chi=0.313 \pm 0.01$. The present outburst also confirms the established general relativistic properties of the system such as the loss of orbital energy to gravitational radiation at the $2 \%$ accuracy level, and it opens up the possibility of testing the black hole no-hair theorem with $10 \%$ accuracy during the present decade.
\end{abstract}

Key words: black hole physics - quasars: general - quasars: individual (OJ 287) - quasars: supermassive black holes

\section{INTRODUCTION}

OJ 287 is recognized as a quasar with roughly 12 year cycles in optical brightness, observed since the 1890s (Sillanpää et al. 1988). Its light curve is definitely not periodic (Hudec et al. 2013), but the deviations from periodicity are systematic and predictable in a model that contains a gravitational-wavedriven inspiraling spinning binary black hole system as its central engine (Valtonen et al. 2008b, 2010b; Byrd et al. 2015). The prediction for the 2015-2016 observing season was that OJ 287 should have a major optical outburst in 2015 December, the brightest optical level in 30 years (see Figure 1 in Valtonen et al. 2011b for the future light curve), coinciding with the centenary of General Relativity. The exact timing of the optical outburst may be used to test predictions of the general relativistic binary black hole model (Valtonen \& Lehto 1997; Valtonen et al. 2010a).

The quasi-periodic pattern of optical outbursts of OJ 287 was explained in 1995 by a model where a secondary black hole in a 12 year orbit impacts the accretion disk of the primary black hole at regular intervals (Lehto \& Valtonen 1996; Sundelius et al. 1997). Owing to the quasi-Keplerian nature of binary black hole orbits in general relativity, the impacts and their associated electromagnetic radiation events cannot occur in a strictly periodic manner (Damour \& Schäfer 1988; Memmesheimer et al. 2004). Attempts to use purely Newtonian orbit models, ignoring post-Newtonian corrections, have failed (Valtonen \& Sillanpää 2011; Valtonen \& Ciprini 2012). ${ }^{59}$

However, it is indeed possible to find a unique mathematical description for the orbit in the post-Newtonian approximation to general relativity, provided a long enough record of past radiation outbursts is available. The solution is sensitive to the loss in gravitational binding energy caused by gravitationalwave emission and the Lense-Thirring effect that forces the binary black hole orbital plane to precess, mainly due to the spin of the primary black hole (Barker \& O'Connell 1975; Damour \& Schäfer 1988).

An essential feature of the model is that the impact outbursts are generated by expanding bubbles of hot gas that have been shocked and pulled out of the accretion disk (see Figure 1). The process is astrophysically rather simple

\footnotetext{
59 For a pictorial depiction of binary black hole orbits in general relativity, see the animations by S. Drasco at www.tapir.caltech.edu/ sdrasco/animations.
}

(Lehto \& Valtonen 1996; Ivanov et al. 1998; Pihajoki 2016), and the resulting radiation emanates from the vicinity of the impact site. Thus, these thermal radiation events are excellent markers for tracing the orbital motion of the secondary around the primary black hole. In contrast, the shocks in jets that also arise as a consequence of the influence of the secondary have a complicated route from cause to effect. These "tidal" outbursts (Sillanpää et al. 1988; Sundelius et al. 1997; Valtonen et al. 2009, 2011a) are also predictable in the binary model, but cannot be used to construct the orbit as accurately as by using the record of the thermal events. In other systems, the regularly repeated events in a jet may be a more likely alternative than thermal events (Ackermann et al. 2015).

\section{OBSERVATIONS}

In anticipation of the predicted outburst, we organized a multisite optical observing campaign aimed at getting photometric and polarimetric data on OJ 287. Both professional astronomical observatories and amateur observers took part in obtaining photometric data from the very beginning of the 2015-2016 season. The telescopes of amateur astronomers are in the 0.20-0.30 m class (Valtonen et al. 2008a).

Photometric observations were carried out by the following observatories: Tuorla Observatory in Finland, Mount Suhora Observatory of the Pedagogical University and Astronomical Observatory of the Jagiellonian University in Poland, University of Athens in Greece, Mount Abu Infrared Observatory in India, and Liverpool Telescope, Kungliga Vetenskapliga Akademien Telescope, Nordic Optical Telescope (NOT), and William Herschel Telescope using the ACAM instrument, in La Palma, Canary Islands, Spain (see Pihajoki et al. 2013 for details). Other telescopes participating were the $0.41 \mathrm{~m}$ PROMPT5 telescope in Chile (Reichart et al. 2005), the $0.6 \mathrm{~m}$ SARA telescope at the Cerro Tololo InterAmerican Observatory, the $0.51 \mathrm{~m}$ reflector in Osaka Kyoiku University, Japan, the $0.25 \mathrm{~m}$ Cassegrain and 0.9/0.6 m Schmidt telescopes of the University Observatory Jena, Germany (Mugrauer \& Berthold 2010; Mugrauer 2016), the $0.77 \mathrm{~m}$ Schmidt Camera of Tonantzintla in Mexico, the $0.60 \mathrm{~m}$ and $1.22 \mathrm{~m}$ reflectors of the Canakkale Onsekiz Mart University Observatory, the $0.60 \mathrm{~m}$ telescope of the University of Adiyaman and the $0.60 \mathrm{~m}$ telescope at the TUBITAK National Observatory, Turkey, and the $0.50 \mathrm{~m}$ robotic telescope at the Ondrejov 
Observatory, Czech Republic. In the continental United States, the photometric data were gathered with the $0.9 \mathrm{~m}$ SARA telescope at Kitt Peak, the $0.40 \mathrm{~m}$ telescope of Florida International University, the $0.76 \mathrm{~m}$ Katzman Automatic Imaging Telescope (KAIT) at the Lick Observatory (Filippenko et al. 2001), the $0.40 \mathrm{~m}$ University of Alabama campus telescope, and the $0.40 \mathrm{~m}$ Arizona State University campus telescope. OJ 287 was measured through the wide-band $R$ filter in most sites. Only the KAIT data were taken without any filter and transformed into the $R$-band. We performed differential photometry on images calibrated for bias, dark, and flatfield with the aperture method. We used GSC 1400-222 $(R=13.74 \mathrm{mag})$ as the comparison star and GSC 1400-444 as the check star.

Measurements with the DIPOL-2 polarimeter (Piirola et al. 2014) installed on the remotely controlled, $0.60 \mathrm{~m}$ telescope at the Haleakala observatory (Tohoku University) were carried out on 13 nights in the interval 2015 November 30-December 15 (UT dates are used throughout this paper). Simultaneous observations in three different passbands $(B, V$, $R$ ) were made by using dichroic beam splitters to divide the light, which was then recorded by three CCDs. On each night, $32 \times 30 \mathrm{~s}$ exposures of OJ 287 were obtained at different orientations (22.5 steps) of the superachromatic half-wave retarder used as the polarization modulator. The fluxes of the target images on the CCD frames were extracted by using a circular aperture of $4^{\prime \prime}-6^{\prime \prime}$ radius.

Polarization and photometry observations of OJ 287 were taken on 20 nights (89 altogether) in the interval 2015 November 28-December 31 with the RINGO3 polarimeter (Arnold et al. 2012) on the fully robotic and autonomous Liverpool Telescope on La Palma, Canary Islands (Steele et al. 2010). Simultaneous observations (120 s duration) in

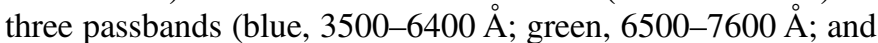
red, 7700-10000 $\AA$ ) were taken using the rapidly rotating (once per $4 \mathrm{~s}$ ) polaroid that modulates the incoming beam of light in 8 rotor positions, and for the photometry the 8 frames are stacked. The beam is simultaneously split by 2 dichroic mirrors into three low-noise electron multiplying CCD cameras. The fluxes of the OJ 287 images on the EMCCD frames were extracted using circular apertures with radii of $4^{\prime \prime}$. By combining the flux from the 8 rotor positions using equations from Clarke \& Neumayer (2002), the linear Stokes parameters were measured and used to calculate the degree and angle of polarization. The data were corrected for the effects of instrumental polarization and depolarization by observation of standard stars from Schmidt et al. (1992).

The polarimetric observations at the $2.5 \mathrm{~m}$ NOT telescope (PI: K.N.) were carried out in the manner described in Valtonen et al. (2009). Polarization observations with the $0.60 \mathrm{~m}$ Cassegrain telescope at Mount Suhora Observatory were carried out during seven nights using four polarimetric filters transmitting light of the polarization planes $0^{\circ}, 45^{\circ}, 90^{\circ}$, and $135^{\circ}$. At least seven full series of measurements in all four filters were performed each night with exposure times between 30 and $90 \mathrm{~s}$, depending on weather conditions and brightness of the target. Fluxes were extracted by using the IRAF "apphot" package. Finally, the degree of polarization and position angles were obtained by the $n$-polarizers method proposed by Sparks \& Axon (1999).

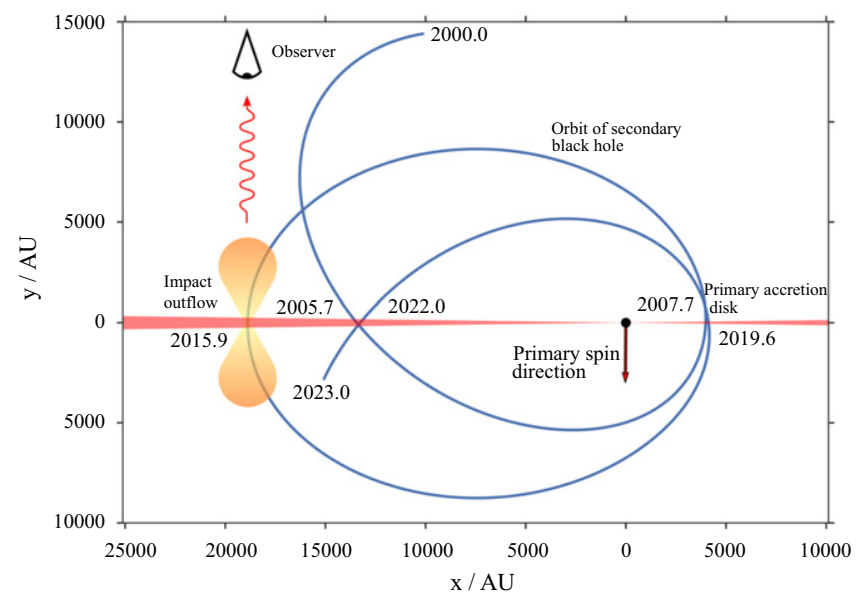

Figure 1. Orbit of the secondary black hole in OJ 287 from 2000 to 2023. The present thermal outburst comes from the disk crossing in 2013 while the nonthermal flux arises from a jet, parallel to the primary spin axis. The next two thermal outbursts are due in 2019 and 2022, following the crossing of the secondary black hole through the accretion disk of the primary black hole.

Two polarimetric observations were obtained using the ARIES Imaging Polarimeter (AIMPOL; Rautela et al. 2004), mounted on the $1.04 \mathrm{~m}$ Sampurnanand Telescope (Sinvhal et al. 1975) at Nainital, India, coupled with a TK $1024 \times 1024$ pixels CCD camera.

A time-domain program for the Swift satellite dedicated to OJ 287 was performed (PI: S.C.) in parallel to these optical multisite observations, using Swift/UVOT filters UVWl, $U V M 2$, and $U V W 2$ and Swift/XRT (0.3-10 keV band). Here, we report primarily the results from the ground-based optical telescope $R$-band and Swift/UVOT $U V W 2$ band where the results were more complete than in other channels, in addition to X-rays. Swift/XRT data were taken in photon-counting mode for a total exposure of about $20 \mathrm{ks}$ divided into daily observations. Each single X-ray spectrum $(0.3-10 \mathrm{keV})$ can be fit by an absorbed single (or broken) power-law model, with an H I column density consistent with the Galactic one in the direction of the source $\left(n_{\mathrm{H}}=2.56 \times 10^{20} \mathrm{~cm}^{-2}\right.$; Kalberla et al. 2005). The X-ray spectra have photon indexes between about 1.4 and 1.9 . The preliminary corresponding unabsorbed $(0.3-2.0 \mathrm{keV})$ integral daily fluxes are reported here, together with simultaneous dereddened UVOT flux-density values obtained with the 3 UV filters.

After starting intensive optical ground-based photometric monitoring of OJ 287 in 2015 September, a series of frames (about 10 images per night) were taken to measure the brightness of the target every clear night. Nightly means were calculated and posted on the campaign's web page. After November 14, a steady rise of the object flux was noticed, and by November 25 it was apparent that it may develop into a major outburst, in the category observed only twice in 12 years. We extended our observations by making them as long as possible each night, measuring also colors at some sites. The source kept brightening very rapidly until it was the brightest it has been in 30 years. After the December 5 maximum, the source declined in stages, until it arrived at its pre-outburst level on December 30 (see Figure 2).

The major outbursts in OJ 287 are recognized by a rapid rise to a narrow peak and then a slower decline with multiple smaller flares. The general shape of the curve in Figure 2 is 


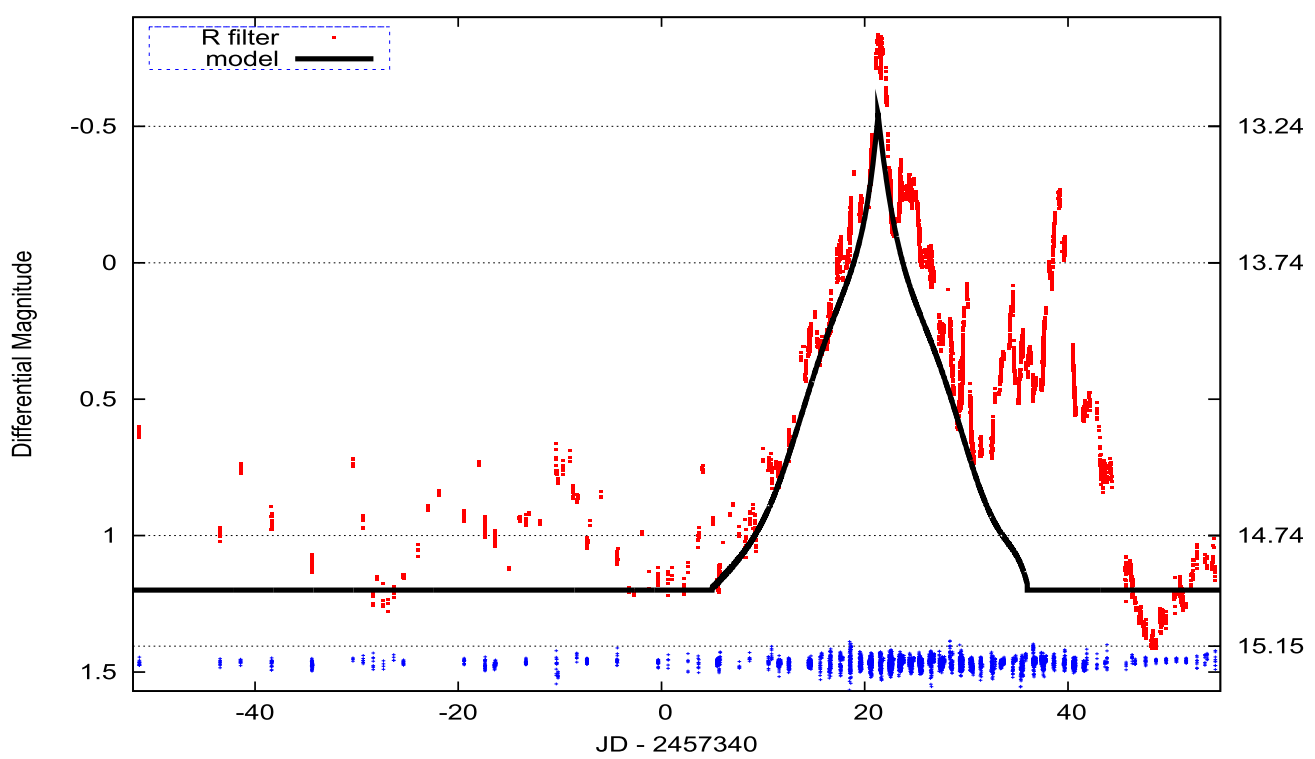

Figure 2. Optical photometry of OJ 287 from 2015 October to December. The optical $R$-band magnitude (squares) is given with respect to the GSC $1400-222$ comparison star. At the bottom (crosses) we show the differences between our comparison and the check star (GSC 1400-444, shifted by 2.05 mag). The theoretical line is explained in the text.

based on a model of a uniform expanding sphere of plasma (Pihajoki 2016). However, as seen generally in observations, e.g., in 1983 (Smith et al. 1987), there is an initial, slowly rising part in the light curve with an additional peak at the maximum. This "standard light curve" (Valtonen et al. 2011b) is used here even though detailed theoretical models do not exist.

The information on the nature of radiation at different stages of the outburst has been limited up to now. In 2007, a good coverage of the outburst was achieved in polarization; it showed that the major component of the outburst was unpolarized, superposed on a lower level of polarized synchrotron emission (Valtonen et al. 2008b). In 1983, the degree of polarization decreased close to zero at the high point of the light curve (Smith et al. 1987). Therefore, we have reason to believe that an underlying unpolarized component, like the curve in Figure 2, also exists in the 2015 outburst, in addition to the usual polarized flares.

Figure 3 shows the evolution of the degree of polarization at different stages of the 2015 outburst. We superpose on the data the expected degree of polarization by using the theoretical line in Figure 2 to separate the thermal and nonthermal components of the outburst. The solid line gives the ratio of the excess radiation above the theoretical line to the total flux, multiplied by 40 . This is what one might expect if the radiation below the theoretical line is unpolarized, as thermal bremsstrahlung should be, and superposed on it we have synchrotron flares with $40 \%$ polarization. The second line assumes an additional $10 \%$ contribution to the degree of polarization from the base level flux. This simple concept seems to work reasonably well. If our separation of the bremsstrahlung from synchrotron flares in the model is correct, then the X-ray emission, coming entirely from the jet, should follow the optical excess emission. The optical excess emission is defined as the total optical flux minus the bremsstrahlung flux, the latter separated from the total flux according to the line in Figure 2.

Figure 4 shows that this is indeed the case. The X-ray flare is rather modest, much smaller than the optical outburst overall, but correlates very well with the excess flare emissions. The flares arising at this time are not different from flares

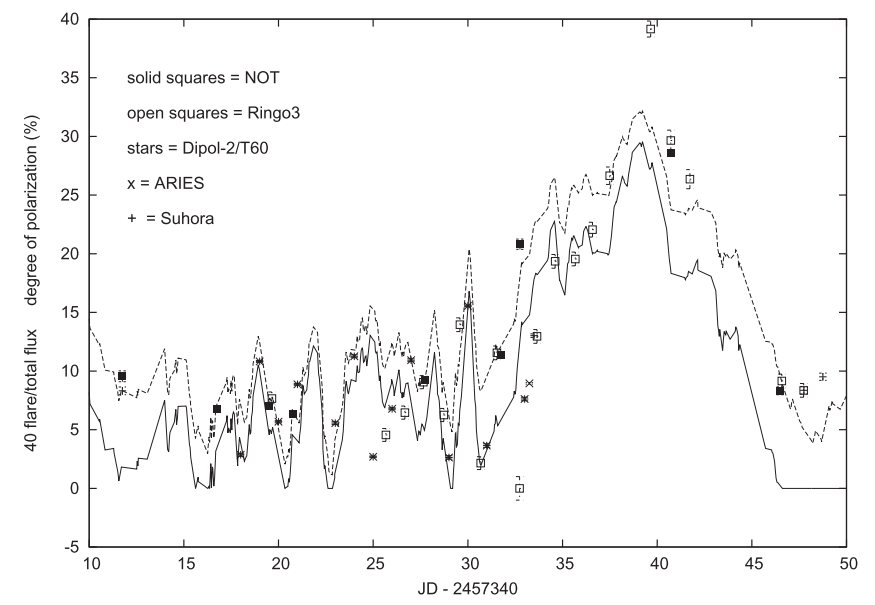

Figure 3. Degree of polarization in the optical $R$-band. The curve represents the expected degree of polarization if the excess nonthermal component, above the line in Figure 2, is $40 \%$ polarized and the rest of the radiation is unpolarized. The dashed line assumes that in addition the base level flux makes a $10 \%$ contribution to the degree of polarization. Nightly median values are plotted for the Ringo3 observations.

observed during the campaigns of the previous 12 months (Edelson et al. 2015). There the X-ray flux was $(4.0 \pm 1) \times 10^{-12} \mathrm{erg} \mathrm{cm}^{-2} \mathrm{~s}^{-1}$, while during our campaign it has been $(4.4 \pm 1) \times 10^{-12} \mathrm{erg} \mathrm{cm}^{-2} \mathrm{~s}^{-1}$, only slightly enhanced.

The UV emission has followed the optical emission rather well in previous campaigns, using a spectral index of 1.35 between the two wavelength ranges (based on data from the Edelson et al. 2015 campaign) of Swift/UVOT. The figure shows that the new line in the $U V W 2$ channel follows the data rather well. Above the thermal components there are the same nonthermal flares that are seen in optical. The Swift UVWI and $U V M 2$ band results are entirely consistent with Figure 4. A more careful study is required to determine the temperature of the bremsstrahlung component at this time (Valtonen et al. 2012). 


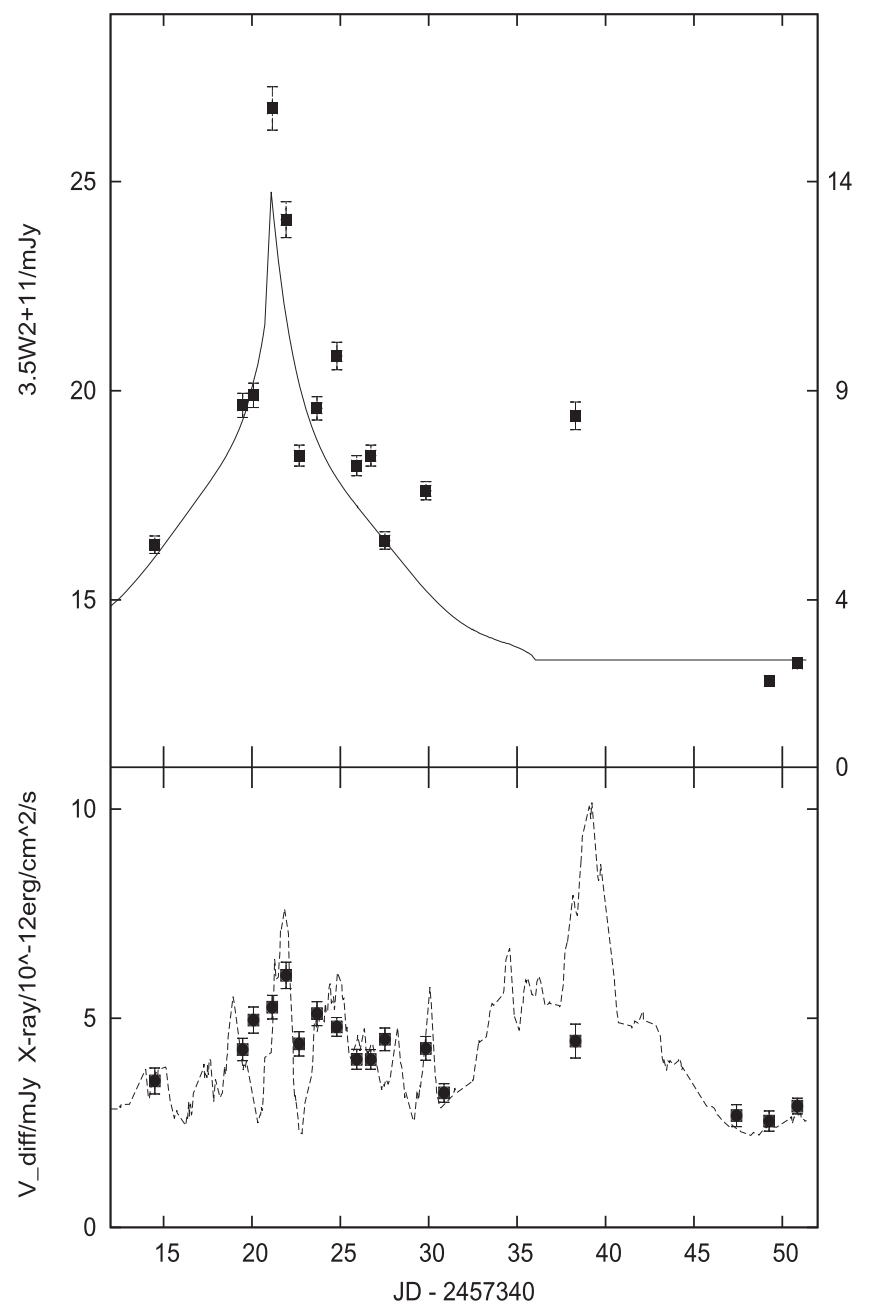

Figure 4. Bottom panel: a comparison of X-ray observations by Swift/XRT in the $0.3-2 \mathrm{keV}$ energy band in $\mathrm{erg} \mathrm{cm}^{-2} \mathrm{~s}^{-1}$ (squares with error bars) with the excess ("jet") emission above the line of Figure 2 (dashed line). Top panel: Swift/UVOT observations in the ultraviolet (UV) UVW2 band (central wavelength $1928 \AA$ ) shown as squares with error bars. The model line shown in Figure 2 has been shifted to the $U V W 2$ band by using the spectral index of 1.35 .

\section{DISCUSSION}

The timing signals are extracted from the optical light curve by identifying the start of the outburst. From Figure 2 it appears that the outburst began on JD 2,457,342.5 \pm 2.5 , which corresponds to the year $2015.874 \pm 0.007$. Using the previously calculated correlation with the spin (Valtonen et al. 2011 b), we get for the Kerr parameter of the primary black hole $\chi=0.313 \pm 0.01(2 \sigma)$. We have checked with orbit solutions, making use of this new timing, that the possible $\chi$ values range between 0.304 and 0.322 . This is a considerable improvement with respect to the previous value $\chi=0.28 \pm 0.08$ (Valtonen et al. 2010b).

For a comparison with black hole spin determinations by X-ray spectroscopy, see Reynolds \& Fabian (2008) and Reynolds (2014). These are based on determining the innermost stable orbit of the accretion disks in Seyfert nuclei or lowredshift quasistellar objects (QSOs) in the radio-quiet realm or in X-ray binaries. Some of the spins are comparable to the spin of OJ 287 , others are close to the maximal value of unity, while the recently observed merger of two black holes produced a spin value of 0.67 (Abbott et al. 2016). In contrast to the X-ray spectroscopy method, in this blazar, we are not dependent on understanding the physics of accretion disks close to the innermost stable orbit; in this sense, the orbital torque method is complementary to X-ray spectroscopy.

The present outburst timing firmly confirms the correctness of the binary black hole central engine model for OJ 287 within its specified parameter ranges, namely, primary mass $(1.83 \pm 0.01) \times 10^{10} M_{\odot}, \quad$ secondary mass $(1.5 \pm 0.1) \times$ $10^{8} M_{\odot}$, and orbital eccentricity (as defined by using the apocentre/pericentre ratio) $0.700 \pm 0.001$.

The present $\chi$ estimate opens up the possibility of measuring the dimensionless quadrupole moment of the primary black hole $\left(q_{2}\right)$ at the $10 \%$ level during the next thermal outburst, predicted to happen in 2019 July (see Figure 1). This should allow one to test the black hole no-hair theorem by verifying the relation $q_{2}=-\chi^{2}$ at that level (Carter 1970; Thorne \& Hartle 1985). However, observing the predicted 2019 July thermal outburst from the Earth will be difficult owing to the proximity of OJ 287 to the Sun at that time.

Additionally, as demonstrated earlier (Valtonen et al. 2010a, 2011b), the occurrence of the outburst within the expected time window confirms the loss of energy by gravitational radiation within $2 \%$ of the prediction by general relativity and is consistent with the no-hair theorem of black holes within an accuracy of $30 \%$. The energy loss from impacts on the accretion disk is four orders of magnitude smaller than the energy loss through gravitational radiation and thus plays no role in the binary model.

Finally, we note that an exceptionally large amount of gas has been pulled away from the primary disk during this impact that occurred close to the apocentre of the binary orbit (Pihajoki et al. 2013, see Figure 1). This gas is expected to feed the two black holes for some time to come and keep OJ 287 active with flares.

The highly polarized $(39 \%$ polarization) flare near JD 2,457,380 is interesting; its degree of polarization is the highest ever measured in OJ 287. The previous record was $36 \%$ polarization measured in the secondary peak of the 1984 major event (Smith et al. 1987). This suggests that the secondary flare is closely connected with the first, unpolarized outburst. One possibility is the activation of the jet of the secondary black hole at these times. The secondary black hole is in the vicinity of the expanding cloud of plasma and will definitely accrete a major part of it-that is, the part that is expanding to its direction. It will be interesting to search for other evidence to associate the secondary flare with the secondary black hole.

In summary, we have shown that the outburst in OJ 287 in 2015 November-December agrees with the binary black hole model, both with regard to the timing and the expected brightness as well as a major outburst component being thermal. The fact that such thermal outbursts are excellent trackers of the secondary black hole orbit allowed us to estimate the spin value of the primary more narrowly than before, $\chi=0.313 \pm 0.01$. This November-December outburst firmly confirms the presence of an inspiraling massive black hole binary in OJ 287. It therefore makes a fitting contribution to general relativity centenary celebrations of 2015-2016 and adds to the excitement over the first direct observation of a transient gravitational-wave signal (Abbott et al. 2016). 
The Swift gamma-ray burst explorer mission is part of NASA's medium explorer (MIDEX) program, led by NASA with participation of Italy and the UK. We would like to thank the Swift team for making these observations possible, in particular D. Malesani as the Swift Observatory Duty Scientist. The authors acknowledge support by the following grants: NCN 2013/09/B/ST9/00599 (S.Z.) and DEC-2011/03/D/ ST9/00656 (M.Z.), GACR 13-33324 S (R.H.), and Academy of Finland grant No. 1274931 (P.P.). A.V.F. and W.-K.Z. are grateful for financial assistance from NSF grant AST1211916, NASA grant NNX12AF12G, the TABASGO Foundation, and the Christopher R. Redlich Fund. We extend our thanks to TUBITAK for partial support in using the T60 telescope with project number 10CT60-76. We are grateful to S. Masda, D. Wagner, T. Zehe, J. Greif, and H. Gilbert for their help in carrying out some of the observations taken at the University Observatory Jena, which is operated by the Astrophysical Institute of the Friedrich-Schiller-University.

KAIT and its ongoing operation were made possible by donations from Sun Microsystems, Inc., the Hewlett-Packard Company, AutoScope Corporation, Lick Observatory, the NSF, the University of California, the Sylvia and Jim Katzman Foundation, and the TABASGO Foundation. Research at Lick Observatory is partially supported by a generous gift from Google.

\section{REFERENCES}

Abbott, B. P., Abbott, R., Abbott, T. D., et al. 2016, ApJL, 818, L22

Ackermann, M., Ajello, M., Albert, A., et al. 2015, ApJL, 813, L41

Arnold, D. M., Steele, I. A., Bates, S. D., Mottram, C. J., \& Smith, R. J. 2012 , Proc. SPIE, 8446, 84462

Barker, B. M., \& O'Connell, R. F. 1975, PhRvD, 12, 329

Byrd, G. G., Chernin, A., Teerikorpi, P., \& Valtonen, M. 2015, in General Relativity: The Most Beautiful of Theories, ed. C. Rovelli (Berlin: De Gruyter \& Co), 67

Carter, B. 1970, PhRvL, 26, 331
Clarke, D., \& Neumayer, D. 2002, A\&A, 383, 360

Damour, T., \& Schäfer, G. 1988, Nuovo Cim, 101, 127

Edelson, R., McHardy, I., Jorstad, S., et al. 2015, ATel, 7056

Filippenko, A. V., Li, W. D., Treffers, R. R., \& Modjaz, M. 2001, in SmallTelescope Astronomy on Global, ed. W. P. Chen, C. Lemme, \& B. Paczyński, Vol. 246 (San Francisco, CA: ASP), 121

Hudec, R., Basta, M., Pihajoki, P., \& Valtonen, M. 2013, A\&A, 559, A20

Ivanov, P. B., Igumenshchev, I. V., \& Novikov, I. D. 1998, ApJ, 507, 131

Kalberla, P. M. W., Burton, W. B., Hartmann, D., et al. 2005, A\&A, 440, 775

Lehto, H. J., \& Valtonen, M. J. 1996, ApJ, 460, 207

Memmesheimer, R.-M., Gopakumar, A., \& Schäfer, G. 2004, PhRvD, 70, 104011

Mugrauer, M. 2016, AN, 337, 226

Mugrauer, M. B., \& Berthold, T. 2010, AN, 331, 449

Pihajoki, P. 2016, MNRAS, 457, 1145

Pihajoki, P., Valtonen, M., Zola, S., et al. 2013, ApJ, 764, 5

Piirola, V., Berdyugin, A., \& Berdyugina, S. 2014, Proc. SPIE, 9147, 8

Rautela, B. S., Joshi, G. C., \& Pandey, J. C. 2004, BASI, 32, 159

Reichart, D., Nysewander, M., Moran, J., et al. 2005, NCimC, 28, 767

Reynolds, C. S. 2014, SSRv, 183, 277

Reynolds, C. S., \& Fabian, A. C. 2008, ApJ, 675, 1048

Schmidt, G. D., Elston, R., \& Lupie, O. L. 1992, AJ, 104, 1563

Sillanpää, A., Haarala, S., Valtonen, M. J., Sundelius, B., \& Byrd, G. G. 1988, ApJ, 325, 628

Sinvhal, S. D., Kandpal, C. D., Mahra, H. S., Joshi, S. C., \& Srivastava, J. B. 1975, oams.conf., 20

Smith, P. S., Balonek, T. J., Elston, R., \& Heckert, P. A. 1987, ApJS, 64, 459

Sparks, W. B., \& Axon, D. J. 1999, PASP, 111, 1298

Steele, I. A., Bates, S. D., Guidorzi, C., et al. 2010, Proc. SPIE, 7735, 773549

Sundelius, B., Wahde, M., Lehto, H. J., \& Valtonen, M. J. 1997, ApJ, 484, 180

Thorne, K. S., \& Hartle, J. B. 1985, PhRvD, 31, 1815

Valtonen, M., \& Ciprini, S. 2012, MmSAI, 83, 219

Valtonen, M., \& Sillanpää, A. 2011, AcPol, 51, 76

Valtonen, M. J., Ciprini, S., \& Lehto, H. J. 2012, MNRAS, 427, 77

Valtonen, M. J., Kidger, M., Lehto, H. J., \& Poyner, G. 2008a, A\&A, 477, 407

Valtonen, M. J., \& Lehto, H. J. 1997, ApJL, 481, L5

Valtonen, M. J., Lehto, H. J., Nilsson, K., et al. 2008b, Natur, 452, 851

Valtonen, M. J., Lehto, H. J., Takalo, L. O., \& Sillanpää, A. 2011a, ApJ, 729,33

Valtonen, M. J., Mikkola, S., Lehto, H. J., et al. 2010a, CeMDA, 106, 235

Valtonen, M. J., Mikkola, S., Lehto, H. J., et al. 2011b, ApJ, 742, 22

Valtonen, M. J., Mikkola, S., Merritt, D., et al. 2010b, ApJ, 709, 725

Valtonen, M. J., Nilsson, K., Villforth, C., et al. 2009, ApJ, 698, 781 